\title{
Energy Aware Distance Vector Routing Scheme for Data Centric Low Power Wireless Sensor Networks
}

\author{
S. Mahlknecht, S. A. Madani and M. Roetzer ${ }^{\dagger}$, \\ ICT / Vienna University of Technology, Vienna, Austria \\ \{mahlknecht,madani\}@ict.tuwien.ac.at \\ †Independent Consultants,Vienna, Austria,matthias.roetzer@aon.at
}

\begin{abstract}
This paper presents Energy Aware Distance Vector Routing (EADV) protocol for ultra low power wireless sensor networks. EADV is based on a simple algorithmic approach to cater for low memory space and low energy available on sensor nodes. EADV assumes a data sink having unrestricted energy. It is inspired from a set of routing protocols including AODV, DSDV, and energy aware routing. The protocol consumes about 128 bytes of RAM. The beauty of EADV is in its computational and implementation simplicity and it keeps network lifetime as primary design parameter.
\end{abstract}

\section{INTRODUCTION}

$\mathrm{T}$ HE driving factors for specialized routing protocols for wireless sensor networks are limited energy resources of sensor nodes, limited processing power and storage, low bandwidth and radio range, unreliable communication channels, variation in available energy, and harsh environment. Routing in sensor network requires a paradigm shift as it poses all together a different set of challenges as compared to traditional routing protocols. For design, factors such as node deployment, data reporting method, node and link heterogeneity, reliability, energy consumption, scalability, network dynamics, connectivity, coverage, data aggregation, and quality of service have to be taken into consideration [1]. In this paper we present Energy Aware Distance Vector Routing protocol (EADV) for ultra low power wireless sensor networks. Complex algorithms are not suited for low power sensor nodes having limited processing power and memory space. EADV, a very simple protocol from computational and implementation perspective is developed to cater for aforementioned constraints. The protocol is inspired from a set of existing protocols including Ad-hoc on demand distance vector routing (AODV), Destination Sequenced Distance Vector Routing (DSDV), and Energy Aware Routing (EAR) [2] - [4].

The network initialization starts with initial broadcast by the sink node with initial broadcast vector. The nodes receiving the initial broadcast vector stores the related information in the routing table and performs initial broadcast forward depending upon conditions discussed later. Once the topology is constructed, routing towards the sink node is performed on the basis of energy reserves of the neighboring nodes and the overall path cost. In contrast to DSDV, AODV, and EAR; an adaptive approach is considered to compute the cost from source node towards the sink node. The algorithm has been proven to work in networks with nodes having less than 256 byte of RAM. Having this much memory only about 128 bytes are available for the routing protocol. The paper is organized as follows. In Section II, we give an overview of related work. In Section III, we provide protocol description. Section IV presents our simulation results. Section V discusses real world implementation. Finally, we conclude in Section VI.

\section{RELATED WORK}

Many research papers are published in recent time to improve the sensor network lifetime on the network layer. Mobile Ad hoc networks (MANET's) are very similar to wireless sensor networks but the routing protocols of MANET's cannot be used for sensor networks for the reasons discussed in [5]. Flooding is a classical way to propagate information and if done blindly, it always results in the broadcast storm problem as discussed in [6]. Gossiping is a probabilistic based flooding scheme, which tries to overcome the broadcast storm problem at the cost of reliability. Sensor Protocol for information via negotiation (SPIN) [7] is a flat data centric routing technique based on exchange of meta-data before actual transmission. The meta-data exchange via data advertisements has proven to be very useful in overcoming the broadcast storm problem including redundancy, overlapping, and resource blindness. However, SPIN does not guarantee information delivery if intermediate nodes between the source and destination are not interested in the data advertisements. Directed Diffusion [8], [9] is an important paradigm for event monitoring sensor networks. It uses attribute value pair for naming the data and queries the sensors in an on demand fashion by using the naming scheme and has achieved many fold energy efficiency as compared to classical flooding techniques but its emphasis on life time of a sensor network is less. The gradient set up phase is also expensive in terms of latency and energy consumption. Moreover, being a query driven data model, Directed Diffusion is not very efficient in applications where information is sent to the sink on continuous basis.

In DSDV [2], an extension of Bellman Ford algorithm, every node maintains a routing table. In DSDV, nodes 
periodically transmit routing table updates generating network traffic. As network size grows, the size of the routing tables and the bandwidth required to update them grows as well. This overhead is considered to be the main weakness of DSDV. AODV [3] is a reactive routing protocol based on DSDV. AODV uses sequence numbers of the destination that results in loop free topologies. Routes are acquired on demand, which results in extra delay known as route acquisition delay. Moreover, a large volume of message overhead is incurred if the routing information is changed e.g. when nodes are moved. Dynamic source routing (DSR) [10] belongs to the same class of protocols and is based on source routing where the source specifies the complete path to be taken by a packet. EAR [4] argues that using the minimum energy path all the times will deplete the node energy on this path and result in a disconnected network topology. EAR instead uses a probabilistic approach in selecting the path to destination by keeping more than one path toward the destination. The problem associated with probabilistic approach is magnified when sensor nodes use energy scavenging techniques for energy. Think of a sensor network with nodes using solar cells for energy. Lets assume one set of nodes is directly under sun light (bright nodes) while the other is not (dark nodes). Now using EAR, there will be a chance of using the dark nodes which have comparatively much less energy than the bright nodes hence resulting in complete drainage of energy from the dark nodes. Rumor routing [1], another variant of directed diffusion uses long lived packets called agents to create paths leading to events. These paths are used, rather than flooding the network, for querying the network. Generally directed diffusion floods the query in the network. Rumor routing queries only such nodes that have observed some particular events rather than querying the entire network. For this purpose, long-lived packets known as agents are used. Rumor routing cannot guarantee the delivery of data and the performance of the protocol heavily depends upon the topology of the network. There are few other routing protocols available including gradient based routing, CADR, COUGAR, and ACQUIRE which are not good for ultra low power sensor networks. A very few of the routing protocols considers simplicity (computational and implementation), pure $\mathrm{N}: 1$ routing and network life time as the primary network design parameters.

\section{PROTOCOL DESCRIPTION}

The protocol is designed for pure sensor networks consisting of a data sink and an arbitrary number of sensor nodes. In comparison to long-hop routing, short multi-hop routing consumes less energy and results in higher throughput or (higher signal to interference ratios) [11]. Our protocol uses the neighbor's and path cost information and relies on short multi-hop routing.

\section{A) Network Initialization}

The data sink issues initialization broadcast called "InitBroadcast" (IB). The IB contains only a few bytes with the source address, packet hop count, and the summed up cost towards the sink node. For the sink node, by definition the last two variables are zero.

\begin{tabular}{|c|c|c|}
\hline \multicolumn{3}{|c|}{ Initial Broadcast Vector } \\
\hline $\begin{array}{c}\text { Source } \\
\text { Address }\end{array}$ & $\begin{array}{c}\text { Hop } \\
\text { Count }\end{array}$ & Cost \\
\hline 8 bit & 8 bit & 8 bit \\
\hline
\end{tabular}

Fig. 1 Initial Broadcast Vector

Fig. 1 shows the frame in more detail containing the socalled "Init Broadcast Vector" (IBV). The sink node is assumed to be a powerful node, which has no energy constraints. This is typically the case, as the sink node will have some wired PC like interface for data logging and analysis.

Every node receiving the IB stores the IBV in its routing table and forwards the broadcast by replacing the source address with its own, incrementing the $\mathrm{HC}$ by 1 and by updating the cost field. We call this process as "Init Broadcast Forward" (IBF).

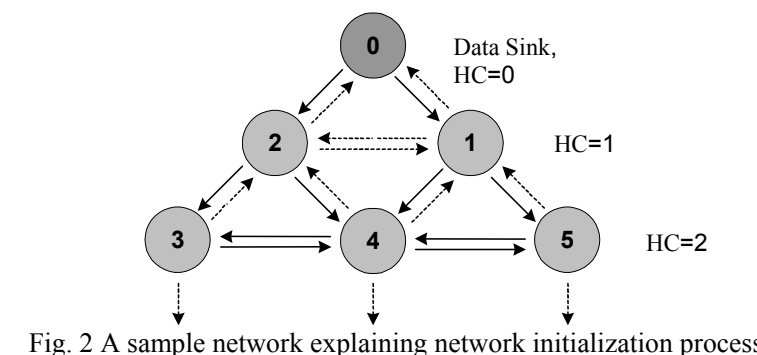

Fig. 2 shows the sequence of the IB messages flooding the network. Each circle represents a sensor node with unique address within a circle. Initially every node is not aware about its own hop count; therefore it sets it to the largest possible value. The continuous line represents those IB packets, which trigger an IBF at the receiving node. The dashed lines represent a transmission of the IBV without consequent IBF at the receiving node. As an example in fig. 1 , if node 1 issues an IBF, nodes $0,2,4$ and 5 receive it. The sink node (address $=0$ ) issues no IBF since by default it forwards no messages, node 2 has a direct connection to the sink node $(\mathrm{HC}=1)$ hence it does not issue an IBF this time but since it also gets an IBV from the data sink with $(\mathrm{HC}=0)$ it issues an IBF triggered by the sink node IB message. For nodes 4 and 5, node 1 is the first connection towards the sink node hence both nodes issue an IBF. Node 4 also gets the IBV from node 2 and since the $\mathrm{HC}$ is lower than its own $\mathrm{HC}$, it issues an IBF message. In this way all nodes at the HC level 2 issues IBF multiple times thus also receiving multiple IBVs with the same $\mathrm{HC}$ as their own hop count. Only when the $\mathrm{HC}$ of 
the IBV gets larger or equal to the $\mathrm{HC}$ of the receiving node no IBF is issued any more. The break condition of termination of a rebroadcast can be stated as follow:

$$
\begin{gathered}
\text { HC IBV } \geq \text { HC Node }+X \\
\text { Where } \quad(0 \leq X \text { and HC Node }>1) \quad \text { as well }
\end{gathered}
$$
as $(X=0$ and $H C$ Node $\leq 1)$. For $X=1$ in the network shown in figure 2 , indicates that lateral communication between nodes of the same $\mathrm{HC}$ can occur. For $X=0_{\text {data is only forwarded to nodes with a smaller }}$ $\mathrm{HC}$ and for $X>1$ reverse path away from the sink node is possible. The larger $\mathrm{X}$ gets, the larger the routing tables become in each node and the more hops towards nodes with higher $\mathrm{HC}$ are possible. For this last option, further measures have to be taken into consideration to avoid circular message routes. This can be avoided by an additional border condition. If $X>1$, no vector will be transmitted in the ACK which contains the actual sending node (next neighbour) as a cheapest path towards the sink node. Instead, the next more expensive vector entry will be used. If there is no additional vector entry available then the ACK includes a Null-Vector whereas the respective entries in the routing tables are deleted and thus the link disconnected. With this mechanism, it can happen that parts of the network will be split off. This is not a big problem because split off network segments will periodically try to issue login broadcasts (LB) in order to register to the network.

To show the importance of the break condition and the necessity of the parameter $X$ one has to envision that cost information in the routing table represents sum of costs along the whole path from the source node to the sink node. Therefore the routing table provides more information than just those about neighbours. But this also means that data can only be sent reverse over that links where the IB came from. Fig. 3 shows an example where the parameter $X$ helps to resolve a specific insufficient network state. For the nodes with the numbers 0 to 6 , the hop count is equal to the node number. For all other nodes the hop count is separated from the number by a comma. If one chooses $X=0$ then a node can only transfer data to a node with lower hop count. $X=1$ means that also connections between nodes of the same hop count are possible and $X>1$ allows reverse paths. When choosing $X=2$, the nodes with the numbers 5 and 6 can transfer data to node number 6 which has a by one higher hop count. Setting $X$ equal to 3 then a node with hop count four can transmit data via nodes that have a by 2 higher hop count because the route and cost information is stored in the routing table during the network initialization.

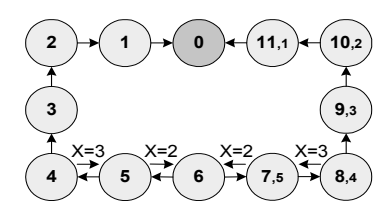

Fig. 3 A topology depicting the importance of parameter $\mathrm{X}$

But why is $X$ necessary? Imagine that node number four is running out of energy. In case of $X=1$, the nodes with number 5 and 6 still transfer there data via node number four until he stops working! In the latter case node number 5 and 6 have to do a LB as soon as they recognize the new network topology. After performing the LB node number 5 and 6 will transfer their data via node number 7 . To avoid a total breakdown of node number 4 , one can choose a higher value for $X$. In the given example $X=3$ is high enough to solve the problem. As soon as the costs for sending data from node 5 via node 4 get more expensive than the route via node 6 , node number 5 will send his data via node 6 . So, choosing an adequate value for $X$ delays the breakdowns of node number four or avoid it in the best case. But a higher value for $X$ leads to large routing tables and long lasting IB, which means that more energy is spent during initialization. Dependent on the type, size and field of application one has to make one's own decision about the value of $X$.

The higher the mobility in the network, the routing tables become inconsistent more frequently. This can only be avoided by periodically issuing initial broadcast (IB) messages from the sink node. The interval can be adapted based on the requirements and characteristics of the network. In typical sensor networks, mobility is very low and mainly consists of nodes being removed or added to the network.

Fig. 4 shows the routing tables for each node. Every entry represents one IBV, with an IBF followed. The left most entry is the next hop address, the next is the number of hops towards the sink node and at the right, the overall cost is shown. Node 3 and node 5 have two routing table entries for node 4, which offers two paths towards the sink node. Based on availability of memory each node can decide whether to store only the lowest cost entry or leaving all entries in the table. In case of keeping only the lowest cost entry for each neighbor, the broadcast can be simplified by only transmitting an IBF if the IBV to be issued is the cheapest entry in the routing table. But just storing the cheapest IBV in the routing table makes it necessary to perform the IB more frequently because otherwise switching to cheaper routes isn't possible!

\section{B) Network Registration:}

A node is registered in an existing network if it is newly added to it or has been offline for a while and did not participate as a routing node in the network. Initially a node sends a "Login Broadcast" (LB). The LB is limited to 
local nodes and is not forwarded by any neighbors of the node to be registered.

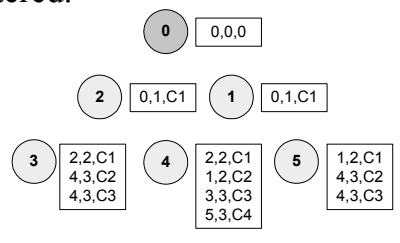

Fig. 4 Routing table of nodes built on basis of IBV and IBF

In the case the network uses a periodic wakeup scheme such as used in CSMA-MPS [12] or WiseMAC [13]; the node has to issue a LB for a longer duration of time in order to reach all nodes that might be in sleep mode for a specific instant of time. In contrast to the IB, the receiver responds to the message with an ACK, which contains the cheapest vector entry of the respective node. All vectors transmitted towards the new node are stored in the routing table. After some time (to be sure it got all ACKs from neighbors) the node issues another broadcast in which the lowest cost vector is contained. The neighbors of the new node store the vector if the conditions in equation 1 are met. With this scheme, it is guaranteed that on one hand new nodes build up their own routing table without flooding the entire network and on the other hand the neighbor nodes are informed of any alternative routes through the newly added neighbors.

\section{C) The Cost Metric}

The network setup as described above includes a cost field in the broadcast messages, which is adapted based on some cost metrics. Cost metrics are important to maximize the lifetime of a network. One has to distinguish between two types of nodes. One type of nodes is the "Battery Supplied Nodes" (BSN), which have an initial amount of energy available, which monotonically decreases over time. Nodes that might be connected to a wired power supply can also be accounted as BSN nodes by giving them a very large amount of energy that never decreases. The other type of nodes are "Energy Scavenging Nodes" (ESN), which typically have only a small buffer of energy such as a capacitor or a small rechargeable battery and obtain their energy from the environment by solar cells, piezo elements or any other sources. These nodes may have a considerable fluctuation of available energy over time, which can cause frequent changes in the actual link costs.

We divide our cost function into three regions; low energy, normal energy and high energy (see Fig. 5). The thresholds for the three regions are defined based on the node's energy reserve and environment. In the normal energy region, we base our cost metric on a quadratic approach while linear and cubic approach is adapted in high and low energy regions. Other possibilities of defining a cost metric are described in [14].

$$
\operatorname{Cos} t=\left\{\begin{array}{ccc}
\left(Q_{\max }-Q\right) & \text { if } & Q \geq 80 \% \\
\left(Q_{\max }-Q\right)^{2} & \text { if } & 10 \% \leq Q<80 \% \\
\left(Q_{\max }-Q\right)^{3} & \text { if } & Q<10 \%
\end{array}\right.
$$

For capacitors as energy storage elements, it is easy to determine Q by measuring the voltage on the capacitor, but for nodes powered by primary batteries this is not a viable approach because it is inaccurate and mainly depends on the type of battery. In the latter case a node could keep track of the activity of the node (how long different units like the CPU or radio are turned on) and estimate the energy consumed in As (Ampere-seconds) which is then subtracted from $Q_{\max }$ to obtain the actual $Q$. In the highenergy region, the cost drops to a constant minimum value, which can also be zero (for instance, if a node is connected to a wired power supply). In the low energy region, the quadratic approach is not enough to cause a single node to become the dominant cost factor on a multi-hop path with many hops. We define therefore a higher order cost function, which lets the cost to increase much quicker as energy levels reach a lower threshold value.

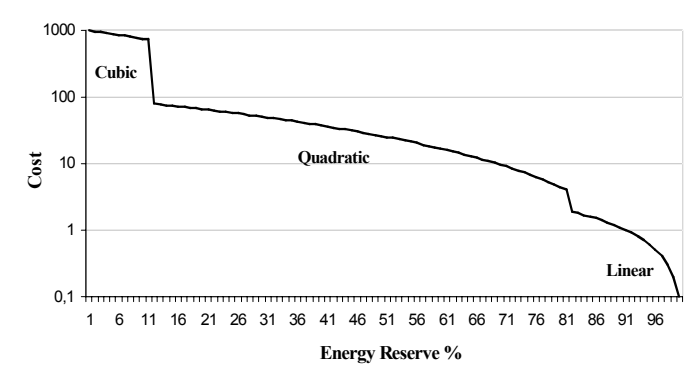

Fig. 5 Behavior of Cost metric against remaining energy of node

In comparison to energy aware routing [4] which chooses the routes based on probabilities, EADV always uses the lowest cost route and adapts costs quickly to maximize network lifetime. The updated cost is included in every acknowledgment packet making dedicated cost update messages dispensable but distributes slowly across the network.

\section{D) Behaviour during operation}

Taking into consideration the network shown in fig. 4 , if node 4 wants to send a sensor value to the sink node, it has four routes at its disposal. It uses the lowest cost route in this case. Assuming the lowest cost route of node 4 is $\mathrm{C} 2$, and then node 4 as well as node 5 will send its data via node 1 hence the energy available at node 1 will decrease faster then the energy from node 2 . To avoid this, the receiving node (in this case node 1) will send an ACK message including the cheapest vector entry out of his routing table. As time goes on the path with the cost $\mathrm{C} 1$ of node 4 over node 2 becomes cheaper then the path over node 1 with the cost $\mathrm{C} 2$. In this case node 4 will try to forward messages over node 2 which will soon update the 
cost $\mathrm{C} 1$. In this way traffic will be balanced between nodes and due to the ACK information costs can be updated quickly which is of special importance when nodes with energy scavenging units are within the network. These nodes have a more dynamic change in available energy where routing tables have to update more frequently.

\section{E) Availability of network topology}

The routing protocol should also allow for reconstructing the topology of the network at the sink node. This is desired in order to visualize the network and is implemented by interleaving packets with routing table updates in between the sensor messages. If a node sends a respective vector called "Topology Packet" (TP) to the sink node after some time when the local table has changed, then the sink node is always informed about topology changes. Not only are the active connections known in these case but also all other available connections. Since network topology changes due to addition and removal of nodes, it is assumed to occur rarely and the energy stored in each node does also change slowly (hourly basis assuming the faster changing energy levels in Ultra-Capacitors), the overhead of routing packets will be limited.

\section{SIMULATION}

The simulation is performed in discrete event simulation system OMNET++. The simulated sensor network has 56 nodes and is distributed in NIT backbone like topology (Fig. 8). Every node is initialized with 10 Watt-Second of energy to reduce the simulation time. The energy utilized for transmission and reception of the data is considered the same and is computed by multiplying voltage, current, and the amount of time depending upon packet size. Other model of energy utilization can be found in [15].

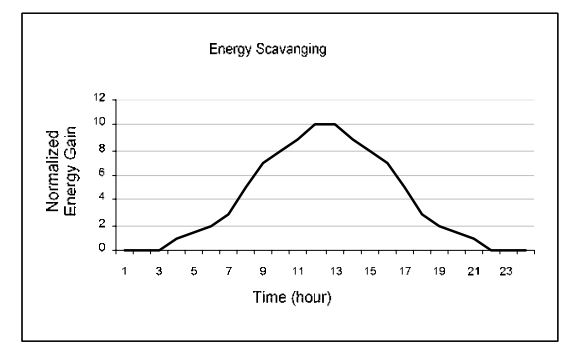

Fig. 6 Energy gain for energy scavenging nodes

For energy scavenging by nodes, bell shaped curve (Fig. 6 ) is considered to compute value of the gained solar energy.

The value obtained is multiplied by probability factor to consider cloudy days and different seasons of the year. The energy utilized by MAC and Application layers is not considered for simplicity. For battery energy drainage, linear discharge model is considered. Other discharge models can be found in [16]. Fig. 7 shows the amount of normalized energy utilized by all the nodes.

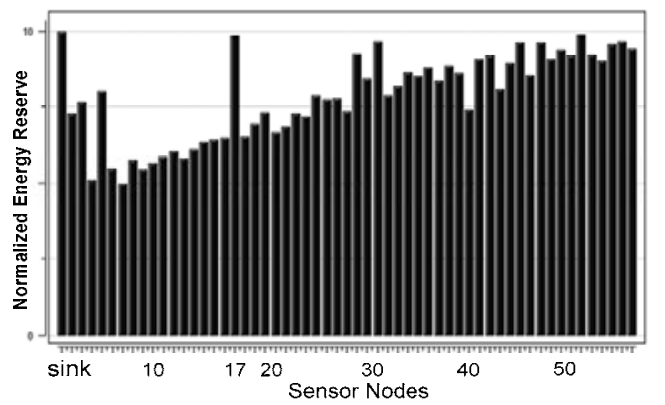

Fig. 7 Remaining normalized energy of nodes after simulation time.

It is clear that the energy utilization is evenly distributed among all the nodes available in the simulated sensor network indicating desired routing, based on energy reserves of the node.

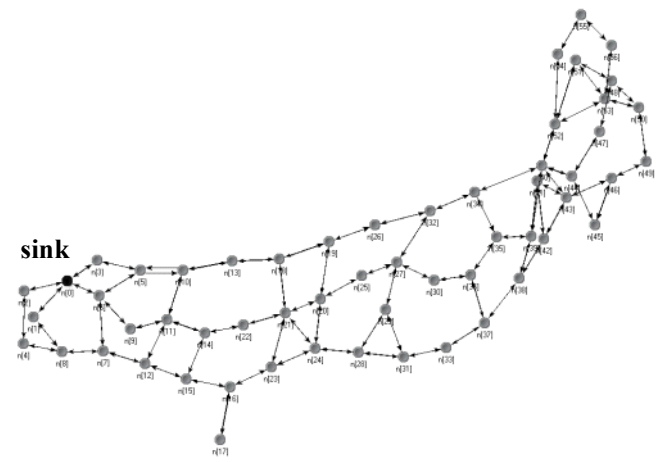

Fig. 8 Sample topology used for simulation

Fig. 7 presents a continuously increasing chart towards the right, which indicates that nodes nearer to sink node (extreme left) are more engaged in forwarding packets. Fig.7 shows the sample topology used for simulation purpose. The few peaks indicates that these nodes are not acting as routing nodes for data, e.g. node 17 in Fig. 7

\section{IMPLEMENTATION}

In the Wireless Self-Sustaining Sensor Network Project [17] (WSSN), at the University of Technology in Vienna, the main research goal was to show that energy-selfsufficient wireless sensor nodes are feasible by using a very efficient overall system implementation with off-theshelf components and efficient protocols. The EADV routing protocol has been implemented on the Tinymote sensor node [18] in a test network of 25 nodes within our office building powered by small solar cells and Ultracapacitors. The network is in operation for many months and despite the winter time and the $100-150 \mu \mathrm{W}$ of average available energy, none of the nodes run out of energy. EADV is supported by a very efficient MAC protocol, the CSMA-MPS [12] optimized for high bit rate radio transceivers [19] and the use of an efficient power overall hardware design. The prototype sensor nodes of very small size are based on the Texas Instruments 
MSP430 ultra low power microcontroller with 256 bytes of RAM and 8Kbytes of Flash memory as well as the high bit rate $2.4 \mathrm{GHz}$ CC2400 radio transceiver from Chipcon. Figure 8 shows a block diagram and picture of the realized sensor node (Tinymote), including a solar-based energy scavenging system.

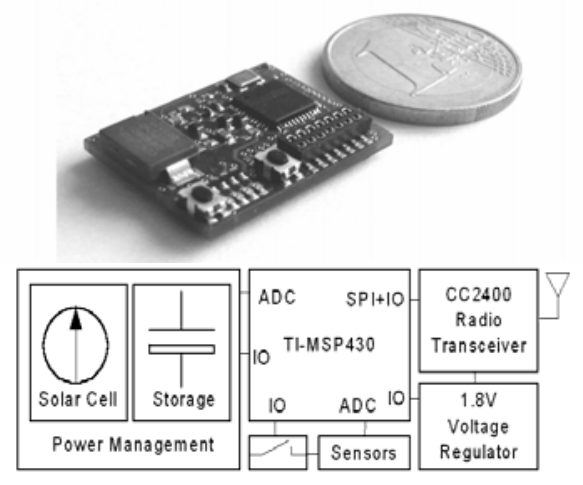

Fig. 8 Sensor node built at ICT, Vienna University of Tech. Austria

EADV fits into less than 128 bytes including routing tables, which optionally could also be stored on the flash memory as the number of neighbor nodes increases. In order to verify the functionality and performance of the protocol in the real world, we implemented the protocol to verify unpredictable energy available at the energy scavenging nodes. Other parameters such as leakage currents at capacitors, shading and interference of the RF signal and detailed modelling of the nodes power consumption made a real world experiment compulsory.

\section{CONCLUSION AND FUTURE WORK}

Based on results obtained from a real world implementation on a low complexity platform, EADV is an energy efficient routing protocol very well suited for long lasting battery-powered or energy scavenging powered sensor nodes with a little amount of network traffic. The simulation of the protocol verifies the correct working of the protocol. Global flooding is kept to a minimum (only during network setup and non-frequent periodical broadcasts by the sink node). The protocol supports many to one communication where every node searches for the lowest cost path towards the closest sink node. A reverse path from the sink node towards sensor nodes is only possible by means of broadcasts. This is not a very limiting restriction because most sensor networks mainly have the specific many to one (sink node) communication pattern.

We intend to extend the protocol with inclusion of location awareness of any node in the network based on techniques using distributed radiolocation algorithms and hardware found in the newest ZigBee based chips [20] from Chipcon. The location estimation algorithm will use received signal strength indicator (RSSI) values from known reference nodes.

\section{REFERENCES}

[1] J.N.Al-Karaki and A. E. Kamal, "Routing Techniques in Wireless Sensor Networks: A Survey", IEEE Wireless Communication, Vol. 11, no. 6,pp.6-28, Dec. 2004.

[2] C. E. Perkins and P. Bhagwat, "Highly dynamic destination sequenced distance vector routing (DSDV) for mobile computers", Comp. Commun. Rev., Oct. 1994, pp. 234-244.

[3] C. Perkins and E. Royer, "Ad hoc On demand distance vector routing", Proceedings of 2nd IEEE Workshop on Mobile Comp. Sys. \& Apps., Feb 1999.

[4] R. C. Shah and J. Rabaey, "Energy Aware Routing for Low Energy Ad Hoc Sensor Networks", IEEE Wireless Communications and Networking Conference (WCNC), March 17-21, 2002, Orlando, FL.

[5] Shijin Dai, Xiaorong Jing, Lemin Li, "Research and Analysis on Routing Protocols for Wireless Sensor Networks", University of Electronic Sciences and Technology of China, 2005.

[6] Sze-Yao Ni, Yu-Chee Tseng, Yuh-Shyan Chen, and Jang-Ping Sheu, The broadcast storm problem in a mobile ad hoc network," in MobiCom '99: Proceedings of the 5th annual ACM/IEEE international conference on Mobile computing and networking. 1999, pp. 151- 162, ACM Press.

[7] W. Heinzelman, J. Kulik, and H. Balakrishnan, "Adaptive protocols for information dissemination in wireless sensor networks," in the Proceedings of the 5th Annual ACM/IEEE International Conference on Mobile Computing and Networking (MobiCom'99), Seattle, WA, August 1999.

[8] C. Intanagonwiwat, R. Govindan and D. Estrin, "Directed diffusion: A scalable and robust communication paradigm for sensor networks", in the Proceedings of the 6th Annual ACM/IEEE International Conference on Mobile Computing and Networking (MobiCom'00), Boston, MA, August 2000.

[9] D. Estrin, et al., "Next century challenges: Scalable Coordination in Sensor Networks," in the Proceedings of the 5th annual ACM/IEEE international conference on Mobile Computing and Networking (MobiCom'99), Seattle, WA, August 1999.

[10] D. B. Johnson and D. A. Maltz, "Dynamic source routing in ad hoc wireless networks", Mobile Computing, Kluwer, 1996, pp. 153-181.

[11] P. Gupta and P. R. Kumar, "The Capacity of Wireless Networks," IEEE Trans. Info. Theory, vol. 46, Mar. 2000, pp. 388-404.

[12] S. Mahlknecht and Rötzer, „CSMA-MPS: A Minimum Preamble Sampling MAC Protocol for Low Power Wireless Sensor Networks", in the proceedings of IEEE WFCS, Vienna, Austria, 2004.

[13] A. El-Hoiydi, "WiseMAC, An Ultra Low Power MAC Protocol for the WiseNET Wireless Sensor Network", ACM SenSys, Los Angeles, 5-7 Nov 2003

[14] Ayad Slhieh, Loren Schwiebert: Power Aware Metrics for Wireless Sensor Networks, Department of ECE, Wayne State University Detroit, IEEE Transactions Vol. 26., No. 4, 2004[12] P. Gupta and P. R. Kumar, "The Capacity of Wireless Networks," IEEE Trans. Info. Theory, vol. 46, Mar. 2000, pp. 388-404.

[15] W. Heinzelman, A.Chandrakasan and H. Balakrishan, "Energy efficient communication protocol for wireless micro-sensor networks", $33^{\text {rd }}$ Hawaii International conference on System Sciences, Jan 2000.

[16] S. Singh, M. Woo, and C.S. Raghavendra. "Power-aware routing in mobile and ad hoc networks". In Proceedings of Fourth Annual ACM/IEEE International Conference on Mobile Computing and Networking (Mobicom), pages181-190, 1998.

[17] S. Mahlknecht, "Energy-Self-Sufficient Wire-less Sensor Networks for Home and Building Environment", Dissertation Thesis at the Institute of Computer Technology, Vienna University of Technology, 2004.

[18] http://www.ict.tuwien.ac.at/wireless/sensornodes.html

[19] S. Mahlknecht and M. Böck, "On the use of High Bit Rate Transceivers for Low Duty Cycle Wireless Sensor Networks", IEEE 7th Africon, Gaborone, Botswana, 2004.

[20] Chipcon Corp. "CC2431 Datasheet Rev. 1.0" www.chipcon.com 